Research Paper

\title{
The optimal strategy of multimodality therapies for resectable gastric cancer: evidence from a network meta-analysis
}

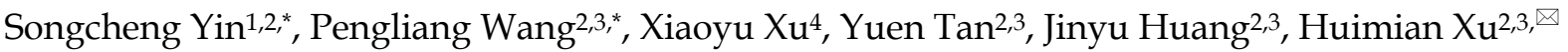 \\ 1. Center for Digestive Disease, The Seventh Affiliated Hospital of Sun Yat-sen University, Shenzhen, China \\ 2. Department of Surgical Oncology, First Affiliated Hospital of China Medical University, Shenyang, China \\ 3. Key Laboratory of Gastric Cancer Molecular Pathology of Liaoning Province, 155 Nanjing North Street, Heping District, Shenyang, China \\ 4. Department of Gynecology, The Seventh Affiliated Hospital of Sun Yat-sen University, Shenzhen, China \\ * These two authors contribute equally in this work
}

$\square$ Corresponding author: Prof. Huimian Xu, Department of Surgical Oncology, First Affiliated Hospital of China Medical University, 155 Nanjing North Street, Heping District, Shenyang, 110001, China. Phone: +86-024-83283556; Fax: +86-024-83283556; E-mail: xuhuimian@126.com

(c) Ivyspring International Publisher. This is an open access article distributed under the terms of the Creative Commons Attribution (CC BY-NC) license (https://creativecommons.org/licenses/by-nc/4.0/). See http://ivyspring.com/terms for full terms and conditions.

Received: 2018.10.06; Accepted: 2019.04.20; Published: 2019.06.02

\begin{abstract}
Background: Controversy continues regarding the optimal strategy of multimodality therapies for resectable gastric cancer. The aim of this network meta-analysis was to determine the efficacy of surgery combined with neoadjuvant or adjuvant chemotherapy $(C T)$, radiotherapy $(R T)$, and chemoradiotherapy (CRT) by integrating the direct and indirect method.

Methods: A systematic search for randomized controlled trials (RCTs) was performed through Medline, Embase, CENTRAL, and PMC databases. Overall survival (OS) was the primary outcome of interest. A Bayesian network meta-analysis was conducted and treatments were ranked based on their effectiveness for improving survival.

Results: Fifty-six RCTs involving 12,435 patients were included. Overall analysis showed that neoadjuvant CRT resulted in a statistically significantly better OS compared with adjuvant CT, adjuvant RT, adjuvant CRT, neoadjuvant $C T$, neoadjuvant RT, and surgery alone. Moreover, subgroup analysis of D2 lymphadenectomy revealed that neoadjuvant CRT was not significant superior to neoadjuvant $C T$ ( $H R=0.67,95 \% \mathrm{Crl} 0.41-1.08$ ), adjuvant CRT (HR $=0.67,95 \% \mathrm{Crl} 0.37-1.21)$, and adjuvant $\mathrm{CT}(\mathrm{HR}=0.60,95 \% \mathrm{Crl} 0.35-1.04)$. With a tendency to survival benefit, neoadjuvant CRT had an $89 \%$ probability of being the best selection.

Conclusions: Our study showed no significant survival advantage for neoadjuvant CRT, though the highest probability of being the best treatment was observed. Further clinical trials are essential to determine the value of neoadjuvant CRT, especially in D2 lymphadenectomy subgroup.
\end{abstract}

Key words: gastric cancer, neoadjuvant therapy, adjuvant therapy, network meta-analysis

\section{Introduction}

Gastric cancer (GC) represents the fifth most prevalent malignancy and the third leading cause of cancer mortality worldwide [1]. Surgery is still the only potential curative treatment, and the 5-year survival rate of patients received curative surgery remains ranging from $24 \%$ to $45 \%$ [2, 3]. Multiple treatment options including surgery-based neoadjuvant and/or adjuvant therapies have been applied to improve the survival of GC patients in the past several decades.
The Southwest Oncology Group (SWOG) 9008/INT-0116 study, the milestone trial of postoperative adjuvant treatment, laid the foundation for adjuvant chemoradiotherapy (CRT), which became a commonly used treatment in the United States [4]. In parts of Europe, perioperative or neoadjuvant chemotherapy (NCT) has become the standard treatment for GC mainly based on the results of the Medical Research Council Adjuvant Gastric Infusional Chemotherapy (MAGIC) trial and 
Federation Nationale des Centres de Luttecontre le Cancer (FNCLCC)/Federation Francophone de Cancerologie Digestive (FFCD) trial $[2,5]$.Thereafter, Adjuvant Chemotherapy trial of S-1 for Gastric Cancer (ACTS-GC) study and the Capecitabine and Oxaliplatin Adjuvant Study in Stomach Cancer (CLASSIC) study established the D2-lymphadenectomy-based surgery combined with adjuvant chemotherapy (CT) as the standard treatment in Asian [6, 7]. Additionally, other treatment options, including adjuvant radiotherapy (RT), neoadjuvant RT and neoadjuvant CRT, have also been applied in GC [8-10].

However, insufficient studies evaluated the comparison between these treatment options and reported conflicting results [11, 12], the comparison among these options remained unknown. Thus, in the current study, we designed a network meta-analysis to evaluate the efficacy of different treatment options in treating resectable GC patients and compared by integrating the previous trials.

\section{Methods}

\section{Literature Search}

Medline, Embase, the Cochrane Central Register of Controlled Trials (CENTRAL), PMC database and the abstracts from the American Society of Clinical Oncology and European Society for Medical Oncology were systematically retrieved up to June 30, 2017. The search strategy consisted of the medical subject headings (MeSH) and key words for GC and treatment options. Additionally, the reference lists from eligible trials and review studies were also screened manually. All the included studies should be in accordance with ethical standards of the responsible committee on human experimentation and the Helsinki Declaration of 1964 and later versions. Two authors (SCY and PLW) conducted the process of the literature search independently. Disagreements were resolved through the group discussion.

\section{Study Selection}

The inclusion criteria for this meta-analysis was performed as follows: (i) prospective randomized controlled trials (RCTs); (ii) patients with pathologically proven as gastric or esophagogastric junction cancer (EGJ); (iii) pair-wise comparison of treatment modalities grouped as surgery combined neoadjuvant/adjuvant treatments (CT, RT, or CRT), and surgery alone; (iv) sufficient data to extract hazard ratios (HRs) to evaluate survival difference; (v) studies published in English. Patients with unresectable, metastatic, or recurrent disease were excluded. If trials with multiple publications were retrieved, the most recent reported one was included to obtain the longest follow-up. We excluded the articles that enrolled esophagogastric junction cancer along with esophageal cancer because of unextracted data of esophagogastric junction cancer alone.

\section{Data Extraction and Quality Assessment}

The following study characteristics were recorded for each included study: (i) basic study information, including the first author, publication year and country; (ii) pathological and demographic characteristics, including gender, age, histopathological type, TNM stage and lymphadenectomy information; (iii) research protocol, including regimens compared, intervention schedules and the number of patients in each group; (iv) outcome indicators, including follow-up information and survival data. All articles were evaluated for risk of bias using the Cochrane Risk of Bias tool, which classified studies into three categories. Two reviewers independently completed data extraction and quality evaluation for each eligible study.

\section{Statistical Analysis}

Overall survival (OS) was selected as the primary endpoint which was measured using hazard ratio (HR) with its 95\% credible intervals (95\% CrIs). Assessment of treatment efficacy were derived first from direct pairwise comparisons in RevMan software version 5.2 for statistical analysis. In order to obtain robust results, the random effects model was performed. Next, a Bayesian network meta-analysis was performed using JAGS and the GeMTC package in $\mathrm{R}$ software (https://drugis.org/ software/rpackages/gemtc). For studies with more than two arms, we considered the correlation between the relative therapeutic effects using the approach reported by Woods [13]. Consistency between direct and indirect evidence was assessed by comparing the pooled HRs from traditional pair-wise comparisons with corresponding results from the network meta-analyses. Moreover, the probability of each treatment being the best was calculated by ranking the relative efficacies of all regimens based on its posterior probabilities. All statistical tests were 2-sided with $a$ of 0.05 .

\section{Results}

\section{Description of the Included Studies}

Fifty-six RCTs contained 12,435 patients were included in our meta-analysis through the systematically literature review (Fig. 1). Among the eligible studies, patients received seven different treatment regimens containing surgery alone or 
surgery-based treatment which involved in adjuvant $\mathrm{CT}$, adjuvant RT, adjuvant CRT, neoadjuvant $\mathrm{CT}$, neoadjuvant RT, and neoadjuvant CRT (Fig. 2). The primary features of the included studies were summarized in Supplementary Table S1. Almost trials were randomized with two comparator arms, in addition to one study comprising three arms involving adjuvant $\mathrm{CT}$, adjuvant $\mathrm{RT}$ and surgery alone [8]. The main clinicopathologic characteristics of the enrolled patients in the included studies were showed in Supplementary Table S2. There was a high degree of consistency among the patients among the studies. The average age of the patients ranged from 55 to 65 years old; and the majority of patients were stage II-II.

\section{Direct Comparison Meta-analysis for OS}

Results of standard pairwise meta-analysis were presented in Supplementary Fig. S1 and S2. Comparing with surgery alone, adjuvant CT (HR = $0.82,95 \%$ CI 0.77-0.87), adjuvant CRT (HR $=0.77,95 \%$ CI $0.65-0.91)$, neoadjuvant CT $(\mathrm{HR}=0.78,95 \% \mathrm{CI}$ 0.68-0.91), neoadjuvant RT $(\mathrm{HR}=0.79,95 \% \mathrm{CI}$ $0.66-0.94)$, and neoadjuvant CRT (HR $=0.35,95 \%$ CI
0.15-0.81) showed significantly OS benefit. Nevertheless, adjuvant RT resulted in a nonsignificant effect on survival ( $\mathrm{HR}=1.17,95 \% \mathrm{CI} 0.92-1.49)$. When compared with adjuvant $\mathrm{CT}$, there was no significantly reduced mortality risk in patients who received adjuvant CRT (HR $=0.91,95 \%$ CI 0.75-1.09) or neoadjuvant CT $(\mathrm{HR}=0.74,95 \%$ CI $0.55-1.00)$, respectively. In addition, no significant difference in OS was found between adjuvant CT and adjuvant RT $(\mathrm{HR}=0.82,95 \%$ CI 0.64-1.05). Similar result was found between neoadjuvant CRT and neoadjuvant CT $(\mathrm{HR}=0.67,95 \%$ CI 0.41-1.08).

\section{Network Meta-analysis for OS}

A random-effect model network meta-analysis was established to compare surgery alone, adjuvant CT, adjuvant RT, adjuvant CRT, neoadjuvant CT, neoadjuvant RT, and neoadjuvant CRT (Fig. 3a). The pooled HRs with 95\% CrIs revealed that all intervention measures resulted in a statistically significantly better OS compared with surgery alone, apart from adjuvant RT $(\mathrm{HR}=1.07,95 \% \mathrm{CrI}$ 0.86-1.34). In the comparisons of adjuvant therapies, efficacy results showed that adjuvant CRT $(\mathrm{HR}=0.71$,

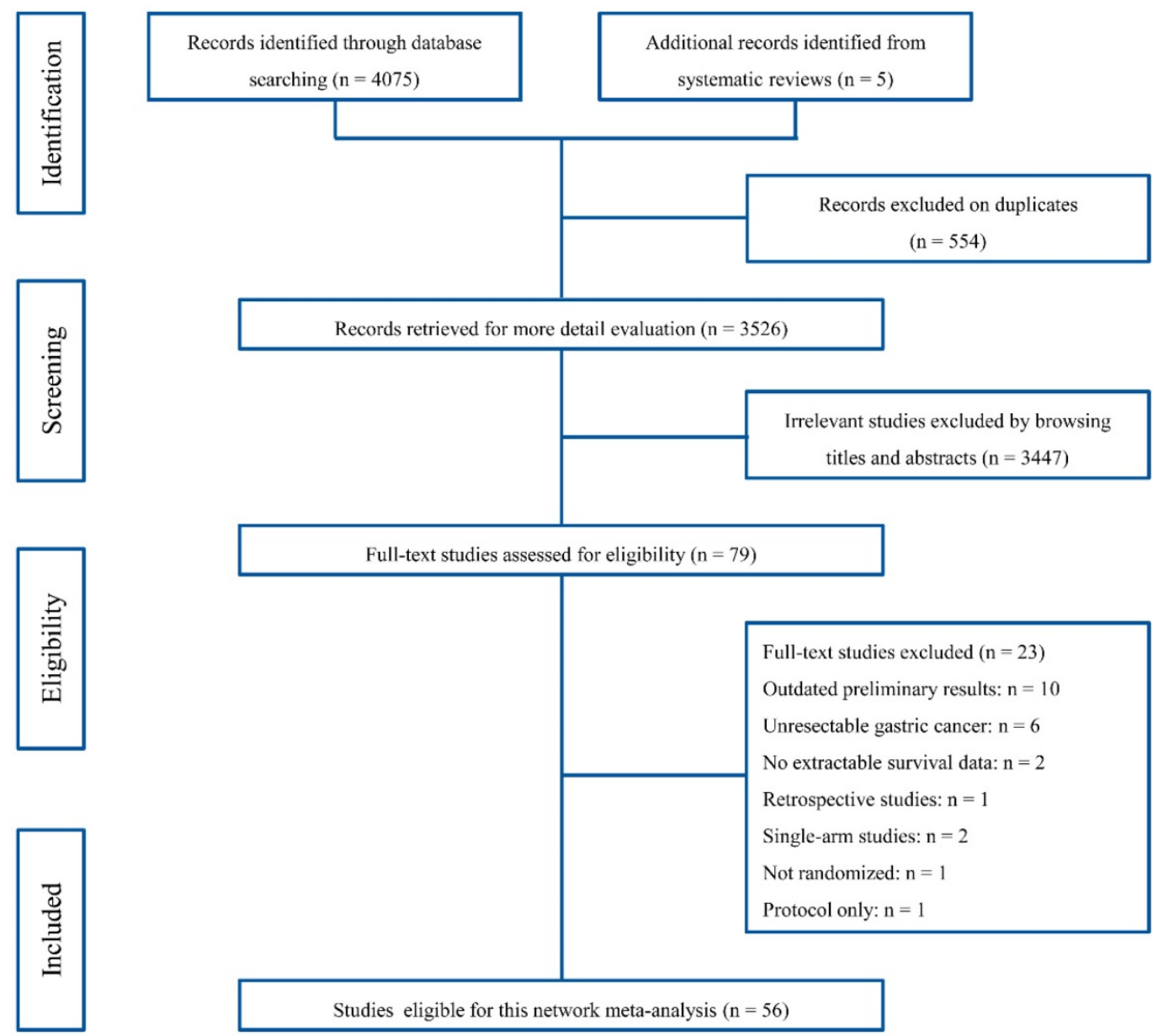

Fig. 1. PRISMA flowchart of the study selection process. 
95\% CrI 0.54-0.93) and CT (HR $=0.78,95 \% \mathrm{CrI}$ 0.62-0.96) were both superior to adjuvant RT. For the comparison between adjuvant CRT and CT, the combined HR did not favour either regimen ( $\mathrm{HR}=$ $0.92,95 \%$ CrI $0.78-1.08)$. With regard to neoadjuvant therapies, patients who underwent neoadjuvant CRT demonstrated a survival advantage compared with those who had neoadjuvant CT ( $\mathrm{HR}=0.61,95 \% \mathrm{CrI}$ $0.39-0.96)$ or RT $(\mathrm{HR}=0.56,95 \%$ CrI $0.33-0.94)$. Moreover, there was no significant difference among the comparisons of adjuvant CT, adjuvant CRT, and neoadjuvant $\mathrm{CT}$.

It is encouraging that neoadjuvant CRT was significant superiority compared with the rest therapies (Fig. 3a). Additionally, the results of the probability of being the most effective therapeutic schedule were as follows: neoadjuvant CRT (97.23\%), neoadjuvant CT $(1.05 \%)$, adjuvant CRT $(1.01 \%)$, neoadjuvant RT $(0.89 \%)$, adjuvant CT (0.01\%), adjuvant RT $(0.01 \%)$, and surgery alone ( $0 \%)$ (Fig. $3 b)$.

\section{Subgroup Analysis}

For patients received D1 lymphadenectomy, adjuvant $\mathrm{CT}$, adjuvant $\mathrm{CRT}$, neoadjuvant $\mathrm{CT}$ and surgery alone were researched in RCTs. Our results showed survival advantage with adjuvant CRT (HR = 0.76, 95\% CrI 0.63-0.91) and neoadjuvant CT (HR = $0.79,95 \%$ CrI 0.65-0.98) compared with surgery alone (Fig. 4a), whereas adjuvant CT had no survival benefit statistically (HR $=0.84,95 \% \mathrm{CrI} 0.69-1.03)$. There was no significant difference between adjuvant CRT and neoadjuvant CT. Ranking analysis revealed that adjuvant CRT, neoadjuvant CT, and adjuvant CT had values at $53.03 \%, 31.89 \%$, and $15.08 \%$ probability of being the best regimen, respectively (Fig. $4 \mathrm{~b}$ ).

In terms of patients underwent D2 lymphadenectomy, adjuvant CT, adjuvant CRT, neoadjuvant $\mathrm{CT}$, neoadjuvant $\mathrm{CRT}$ and surgery alone were applied in RCTs. These treatment options were all associated with a survival advantage over surgery alone (Fig. 4c). Neoadjuvant CRT tended to be superior to neoadjuvant $\mathrm{CT}(\mathrm{HR}=0.67,95 \% \mathrm{CrI}$ $0.41-1.08)$, adjuvant CRT $(\mathrm{HR}=0.67,95 \% \mathrm{CrI}$ $0.37-1.21)$, and adjuvant $\mathrm{CT}(\mathrm{HR}=0.60,95 \% \mathrm{CrI}$ 0.35-1.04) but with nonsignificant. Additionally, neoadjuvant CRT had the highest (89.05\%) probability of being the best treatment (Fig. $4 \mathrm{~d}$ ).

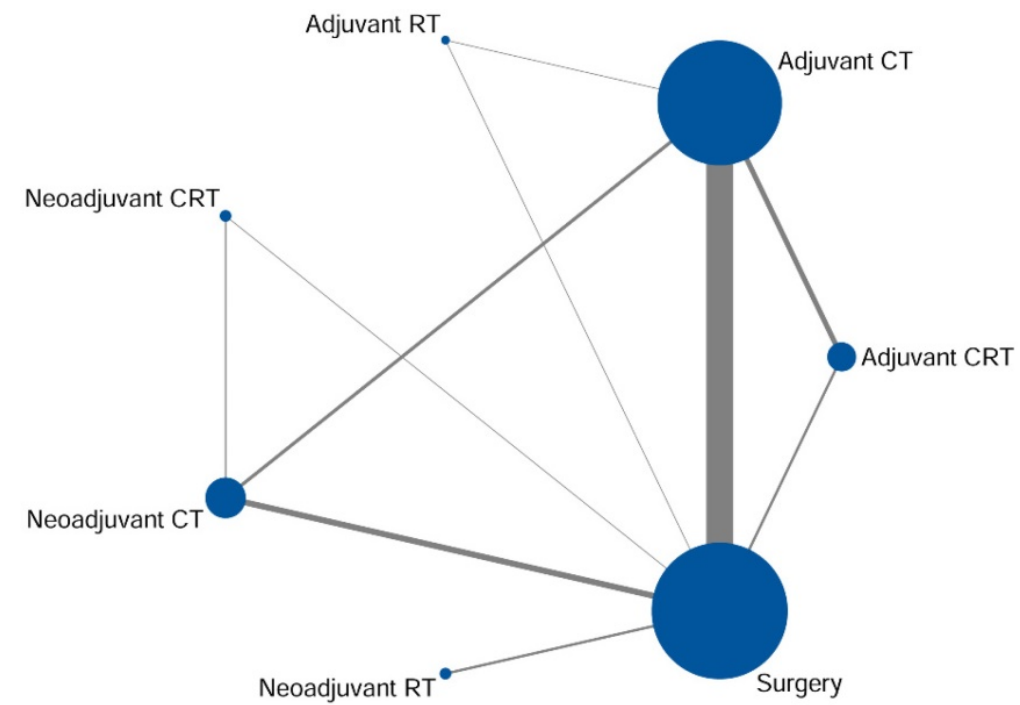

Fig. 2. Network of eligible comparisons for network meta-analysis. The size of each node corresponds to the number of eligible subjects and the line thickness reflects the number of trials for each comparison. CT adjuvant chemotherapy, RT radiotherapy, CRT chemoradiotherapy

A

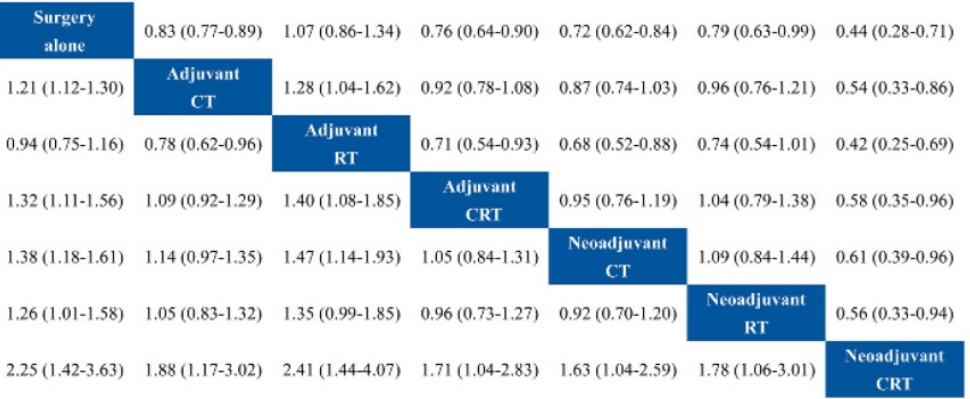

B

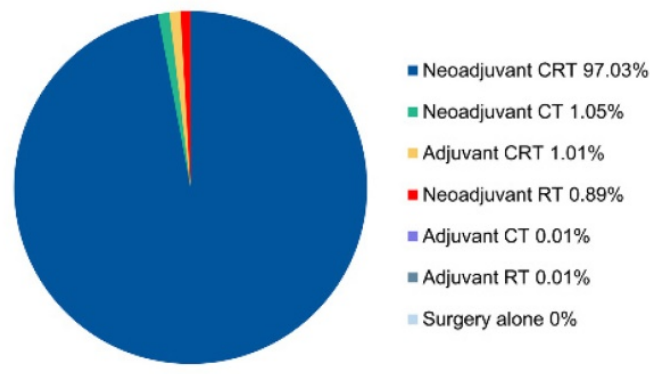

Fig. 3. Results ofentire network meta-analysis for overall survival. (a) Pooled hazard ratios and $95 \%$ credible intervals from network meta-analysis. (b) Probability of being the best treatment. CT adjuvant chemotherapy, RT radiotherapy, CRT chemoradiotherapy 
A

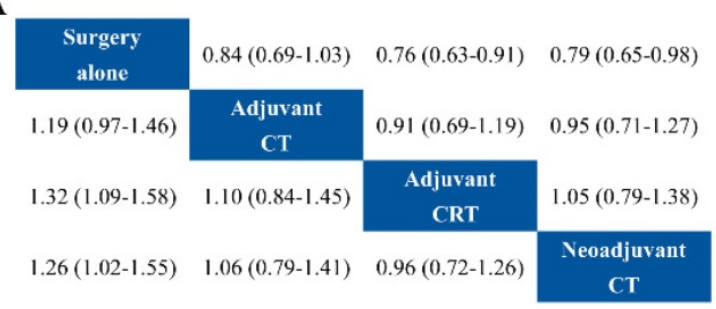

C

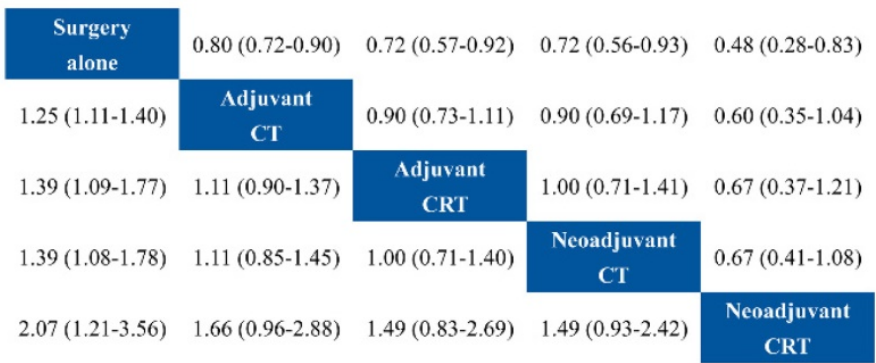

B

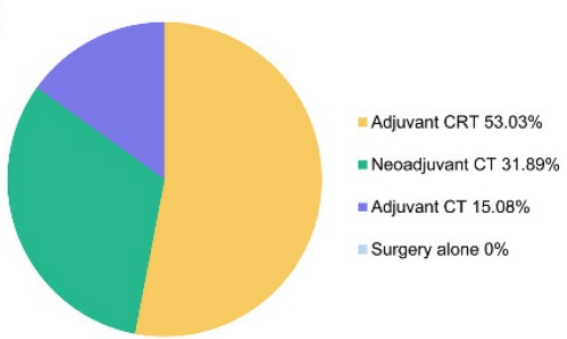

D

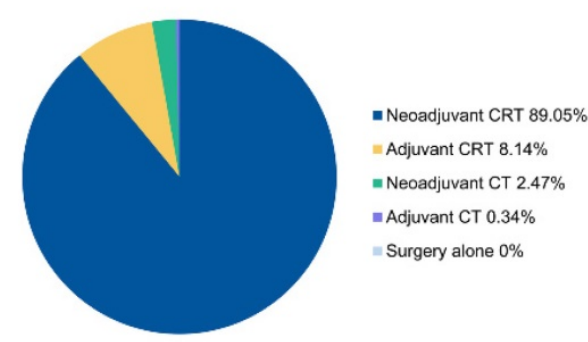

Fig. 4. Results of subgroup analysis for overall survival. Relative effects in combined hazard ratios and $95 \%$ credible intervals in subgroup of D1 (a) and D2 (c) lymphadenectomy. Probability of being the best treatment on overall survival in in subgroup of D1 (b) and D2 (d) lymphadenectomy. CT adjuvant chemotherapy, RT radiotherapy, CRT chemoradiotherapy

Table 1. Major Toxic effects (grade 3 and above)

\begin{tabular}{lllllll}
\hline Treatment strategy & $\begin{array}{l}\text { No. of trials } \\
\text { (patients) }\end{array}$ & \multicolumn{2}{l}{ Number of patients (\%) } \\
\cline { 2 - 6 } & Nausea/vomiting & Diarrhea & Anemia & Leukopenia & Thrombocytopenia & Stomatitis/Mucositis \\
\hline Adjuvant CT & $23(3277)$ & 12.8 & 6.2 & 5.9 & 17.8 & 4.5 \\
Adjuvant CRT & $5(549)$ & 9.7 & 1.6 & 1.6 & 30.0 & 1.9 \\
Neoadjuvant CT & $4(422)$ & 9.7 & 2.8 & 4.7 & 11.6 & 2.4 \\
Neoadjuvant CRT & $1(60)$ & N/A & N/A & N/A & 11.7 & 5.5 \\
\hline
\end{tabular}

Abbreviations: CT, chemotherapy; RT, radiotherapy; CRT, chemoradiotherapy; N/A, not available

\section{Comparison of toxicity}

The grade 3 and above adverse events data from 27 RCTs were showed in Table 1 . In general, there was no obviously difference in the toxicity for patients treated with adjuvant CT, adjuvant CRT, neoadjuvant $\mathrm{CT}$, and neoadjuvant CRT. For patients who received adjuvant CT had the highest rate of diarrhea (6.2\%) when compared with adjuvant CRT, neoadjuvant CT, and neoadjuvant CRT. The incidence of leukopenia $(30.0 \%)$ was the highest among the patients with adjuvant CRT. Additionally, only one trial reported the toxicity data for neoadjuvant CRT. And the most common adverse events were leukopenia (11.7\%) and Thrombocytopenia (5.0\%).

\section{Quality Assessment and Network Consistency}

Minimal bias was found among majority of studies by using the Cochrane risk of bias tool. Risk of bias summary by domain and each study was showed in Supplementary Fig. S3. No studies showed high risk bias in the integrity of outcome data and comprehensiveness of the report, which was impersonal and had a relatively low bias.

We evaluated the consistency between direct and indirect comparisons with node-split models and the results showed that no significant inconsistency for OS (all $P>0.05$, Supplementary Fig. S4).

\section{Discussion}

Evidence-based multiple effective treatment modalities were applied in the treatment of resectable GC and the prognosis of patients were also improved during the past decades. However, the comparison of these treatment options remained unclear. To our knowledge, this is the first study to synthesize all efficacy evidence from 56 trials, compared different treatment options using a Bayesian network meta-analysis. The results showed that neoadjuvant CRT resulted in a statistically significantly better OS compared with adjuvant CT, adjuvant RT, adjuvant CRT, neoadjuvant $\mathrm{CT}$, neoadjuvant $\mathrm{RT}$, and surgery alone. Subgroup analysis revealed that neoadjuvant CRT tended to be superior to adjuvant CT, adjuvant CRT, and neoadjuvant CT which had the highest probability of being the best treatment and might be the best treatment strategy on the basis of D2 lymphadenectomy.

D2 lymphadenectomy, accepted as the standard operation for advanced GC in both Asian and Western, is associated with lower loco-regional 
recurrence and cancer-specific death [14]. Actually, surgery alone cannot achieve a biological cure for advanced GC, even though the extended lymphadenectomy is performed. Adjuvant and neoadjuvant therapies are generally accepted to eradicate microscopic disease, result an additional $10 \%-15 \%$ survival benefit based on the curative surgical resection [15].

Adjuvant CRT gained much popularity in the United States since the results of INT-0116 study reported [4]. Thereafter, D2 lymphadenectomy combined with adjuvant $\mathrm{CT}$ has become the standard treatment in Asia mainly based on the ACTS-GC and CLASSIC trials [6, 7]. Our results also found that adjuvant CRT and CT were superior to surgery alone. These results confirmed the necessity of postoperative adjuvant treatment. For adjuvant RT, non-significant result was observed when compared with surgery alone. Additionally, the comparison between adjuvant CRT and adjuvant CT also showed non-significant results. Subgroup analysis showed that adjuvant CRT was superior to surgery alone but positive results were not found in adjuvant CT for patients with D1 lymphadenectomy. Several previous studies also showed that the advantages of adjuvant or additional RT could be observed in patients with lymph nodes metastasis, D1 resections and positive margins [16-18]. These results elucidated that postoperative RT may serve as a supplementary treatment option when combined with adjuvant CT.

The superiority of neoadjuvant CT mainly attributes to the increasing rate of $\mathrm{R} 0$ resection and controlling microscopic disease. For the comparison between neoadjuvant $\mathrm{CT}$ and adjuvant $\mathrm{CT}$, the results of our direct comparison and indirect comparison analyses indicated insignificantly difference, which was consistent with previous study [19]. However, no clinical trials evaluated the difference between neoadjuvant CT and adjuvant CRT. Recently, a retrospective study showed that patient received neoadjuvant CT was superior to those received adjuvant CRT in terms of survival [20]. Nevertheless, our current study did not obtain the same results and further study will be needed to evaluate these two treatment options.

Notably, the overall analysis indicated that neoadjuvant CRT was significantly superior to other therapeutic strategy in terms of the rank probability of being the best selection. However, subgroup analysis of D2 lymphadenectomy showed that patients with neoadjuvant CRT seemed to be correlated with a better but a nonsignificant survival advantage. With the highest probability of being the best treatment, the level of evidence for neoadjuvant CRT was downgraded from high to moderate, as the CrI crossed the unit [21]. Since the tumor has not changed the anatomical location and is rich in vascularization before surgery, the neoadjuvant CRT is more effective and sensitive. Moreover, smaller irradiated volumes and therefore less toxic reactions were observed lead to well tolerant for patients received preoperative RT [15]. Thus, more feasibility and safety neoadjuvant CRT resulted in higher rate of $\mathrm{pCR}$ and $\mathrm{R} 0$ resection [22-24]. In the ongoing prospective trials, TOPGEAR trial was designed to compare neoadjuvant CRT with neoadjuvant CT followed by postoperative CT with D2 lymphadenectomy. Until this result is available, our data presented here providing an evidence of support neoadjuvant CRT.

The phase III MAGIC and FNLCC/FFCD trials confirmed that perioperative CT was superior to surgery alone and was widely used in Europe $[2,5]$. Unfortunately, fewer than $50 \%$ patients completed the postoperative chemotherapy in these two trials, mainly because of the surgical complications and nutrition status [5]. Even though the higher R0 resection and $\mathrm{pCR}$ rates were achieved, unsatisfied recurrence rate, especially the distant recurrence rate, were obtained in patients received neoadjuvant CT/CRT $[2,5,10,25]$. A previous retrospective study reported that postoperative $\mathrm{CT}$ gained more survival benefit than observation for patients received neoadjuvant CRT [26]. This result further confirmed the superiority of the perioperative treatment pattern. Future trials should consider the difference and evaluate the neoadjuvant and perioperative treatment properly.

Moreover, tumor location may also affect the treatment options. A study investigated the treatment trend in the United States showed that noncardia GC patients had different treatment pattern compared with cardia patients. Preoperative treatment, especially the preoperative CRT, was the most commonly used treatment among cardia patients, whereas the postoperative treatment was the most commonly used among noncardia patients [27]. Additionally, the molecular heterogeneity and subtypes had been explored in different tumor location and this might also affect the response to the chemotherapy and radiotherapy [28, 29]. Thus, the effect of the tumor location and treatment response should be considered in the future trials.

Several limitations should be acknowledged in present study. The trials included in our study lasted for a long period and the improvement of treatment schedules or technique may affect our findings. We evaluated the consistency with node-split models and showed relatively well transitivity. Moreover, our study used all information from published data other than individual patient data, which might influence 
the accuracy of assessment, even though we obtained part of information from previously individual patient data meta-analysis [30]. Additionally, some certain endpoints like disease-free survival and disease-specific survival could not obtain from published studies, which might influence our analysis. Several studies investigated the treatment both in esophagogastric junction cancer and gastric cancer. However, part of trials could not obtain the subgroup data for these two tumor sites. Thus, we may not evaluate the effects of treatment in different tumor location. Furthermore, it was difficult to implement subgroup analysis stratified by different regions (Asia, Europe and the US), because studies in different areas were inadequate. Most Asian and European trials used D2 lymph node dissection, while the US studies focused on D1 lymph node dissection. Therefore, subgroup analysis of lymph node dissection may partly reflect the subgroup results in different regions.

\section{Conclusions}

Our findings showed a potential survival advantage conferred by neoadjuvant CRT. Despite nonsignificant survival advantage was observed for patients with D2 lymphadenectomy, neoadjuvant CRT had the highest probability of being the best treatment. Future direct head-to-head clinical trials are needed to identify the effectiveness of neoadjuvant CRT in D2 lymphadenectomy subgroup.

\section{Supplementary Material}

Supplementary figures and tables. http://www.jcancer.org/v10p3094s1.pdf

\section{Acknowledgments}

This work was supported by National Natural Science Foundation of China (No.81772549 and 81572334 to HMX, No.81602522 to JYH). The funders had no role in study design, data collection and analysis, decision to publish, or preparation of the manuscript.

\section{Ethical Standards}

All procedures performed were in accordance with the ethical standards of the responsible committee on human experimentation (institutional and national) and with the Helsinki Declaration of 1964 and later versions. Informed consent or an appropriate substitute was obtained from all patients prior to inclusion in the study.

\section{Competing Interests}

The authors have declared that no competing interest exists.

\section{References}

1. Torre LA, Bray F, Siegel RL, Ferlay J, Lortet-Tieulent J, Jemal A. Global cancer statistics, 2012. CA: a cancer journal for clinicians. 2015; 65: 87-108.

2. Ychou M, Boige V, Pignon JP, Conroy T, Bouche O, Lebreton G, et al. Perioperative chemotherapy compared with surgery alone for resectable gastroesophageal adenocarcinoma: an FNCLCC and FFCD multicenter phase III trial. Journal of clinical oncology: official journal of the American Society of Clinical Oncology. 2011; 29: 1715-21.

3. Bonenkamp JJ, Hermans I, Sasako M, van de Velde CJ, Welvaart K, Songun I, et al. Extended lymph-node dissection for gastric cancer. The New England journal of medicine. 1999; 340: 908-14.

4. Macdonald IS, Smalley SR, Benedetti I, Hundahl SA, Estes NC, Stemmermann $\mathrm{GN}$, et al. Chemoradiotherapy after surgery compared with surgery alone for adenocarcinoma of the stomach or gastroesophageal junction. The New England journal of medicine. 2001; 345: 725-30.

5. Cunningham D, Allum WH, Stenning SP, Thompson JN, Van de Velde CJ, Nicolson $\mathrm{M}$, et al. Perioperative chemotherapy versus surgery alone for resectable gastroesophageal cancer. The New England journal of medicine. 2006; 355: 11-20.

6. Sakuramoto S, Sasako M, Yamaguchi T, Kinoshita T, Fujii M, Nashimoto A, et al. Adjuvant chemotherapy for gastric cancer with S-1, an oral fluoropyrimidine. The New England journal of medicine. 2007; 357: 1810-20.

7. Bang YJ, Kim YW, Yang HK, Chung HC, Park YK, Lee KH, et al. Adjuvant capecitabine and oxaliplatin for gastric cancer after D2 gastrectomy (CLASSIC): a phase 3 open-label, randomised controlled trial. Lancet. 2012; 379: 315-21.

8. Hallissey MT, Dunn JA, Ward LC, Allum WH. The second British Stomach Cancer Group trial of adjuvant radiotherapy or chemotherapy in resectable gastric cancer: five-year follow-up. Lancet. 1994; 343: 1309-12.

9. Zhang ZX, Gu XZ, Yin WB, Huang GJ, Zhang DW, Zhang RG. Randomized clinical trial on the combination of preoperative irradiation and surgery in the treatment of adenocarcinoma of gastric cardia (AGC)--report on 370 patients. International journal of radiation oncology, biology, physics. 1998; 42: 929-34.

10. Stahl M, Walz MK, Stuschke M, Lehmann N, Meyer HJ, Riera-Knorrenschild J, et al. Phase III comparison of preoperative chemotherapy compared with chemoradiotherapy in patients with locally advanced adenocarcinoma of the esophagogastric junction. Journal of clinical oncology: official journal of the American Society of Clinical Oncology. 2009; 27: 851-6.

11. Park SH, Sohn TS, Lee J, Lim DH, Hong ME, Kim KM, et al. Phase III Trial to Compare Adjuvant Chemotherapy With Capecitabine and Cisplatin Versus Concurrent Chemoradiotherapy in Gastric Cancer: Final Report of the Adjuvant Chemoradiotherapy in Stomach Tumors Trial, Including Survival and Subset Analyses. Journal of clinical oncology: official journal of the American Society of Clinical Oncology. 2015; 33: 3130-6.

12. Zhu WG, Xua DF, Pu J, Zong CD, Li T, Tao GZ, et al. A randomized, controlled, multicenter study comparing intensity-modulated radiotherapy plus concurrent chemotherapy with chemotherapy alone in gastric cancer patients with D2 resection. Radiotherapy and oncology: journal of the European Society for Therapeutic Radiology and Oncology. 2012; 104: 361-6.

13. Woods BS, Hawkins N, Scott DA. Network meta-analysis on the log-hazard scale, combining count and hazard ratio statistics accounting for multi-arm trials: a tutorial. BMC medical research methodology. 2010; 10: 54-62.

14. Songun I, Putter H, Kranenbarg EM, Sasako M, van de Velde CJ. Surgical treatment of gastric cancer: 15-year follow-up results of the randomised nationwide Dutch D1D2 trial. The Lancet Oncology. 2010; 11: 439-49.

15. Van Cutsem E, Sagaert X, Topal B, Haustermans K, Prenen H. Gastric cancer. Lancet. 2016: 388: 2654-64.

16. Lee J, Lim DH, Kim S, Park SH, Park JO, Park YS, et al. Phase III trial comparing capecitabine plus cisplatin versus capecitabine plus cisplatin with concurrent capecitabine radiotherapy in completely resected gastric cancer with D2 lymph node dissection: the ARTIST trial. Journal of clinical oncology: official journal of the American Society of Clinical Oncology. 2012; 30: 268-73.

17. Dikken JL, Jansen EP, Cats A, Bakker B, Hartorink HH, Kranenbarg EM, et al. Impact of the extent of surgery and postoperative chemoradiotherapy on recurrence patterns in gastric cancer. Journal of clinical oncology: official journal of the American Society of Clinical Oncology. 2010; 28: 2430-6.

18. Stumpf PK, Amini A, Jones BL, Koshy M, Sher DJ, Lieu CH, et al. Adjuvant radiotherapy improves overall survival in patients with resected gastric adenocarcinoma: A National Cancer Data Base analysis. Cancer. 2017; 123: 3402-9.

19. Yang Y, Yin X, Sheng L, Xu S, Dong L, Liu L. Perioperative chemotherapy more of a benefit for overall survival than adjuvant chemotherapy for operable gastric cancer: an updated Meta-analysis. Scientific reports. 2015; 5: 12850.

20. Fitzgerald TL, Efird JT, Bellamy N, Russo SM, Jindal C, Mosquera C, et al. Perioperative chemotherapy versus postoperative chemoradiotherapy in patients with resectable gastric/gastroesophageal junction adenocarcinomas: A survival analysis of 5058 patients. Cancer. 2017; 123: 2909-17.

21. Salanti G, Del Giovane C, Chaimani A, Caldwell DM, Higgins JP. Evaluating the quality of evidence from a network meta-analysis. PloS one. 2014; 9: e99682.

22. Ajani JA, Winter K, Okawara GS, Donohue JH, Pisters PW, Crane $\mathrm{CH}$, et al. Phase II trial of preoperative chemoradiation in patients with localized gastric adenocarcinoma (RTOG 9904): quality of combined modality therapy and 
pathologic response. Journal of clinical oncology: official journal of the American Society of Clinical Oncology. 2006; 24: 3953-8.

23. Rivera F, Galan M, Tabernero J, Cervantes A, Vega-Villegas ME, Gallego J, et al. Phase II trial of preoperative irinotecan-cisplatin followed by concurrent irinotecan-cisplatin and radiotherapy for resectable locally advanced gastric and esophagogastric junction adenocarcinoma. International journal of radiation oncology, biology, physics. 2009; 75: 1430-6.

24. Pera M, Gallego R, Montagut C, Martin-Richard M, Iglesias M, Conill C, et al. Phase II trial of preoperative chemoradiotherapy with oxaliplatin, cisplatin, and 5-FU in locally advanced esophageal and gastric cancer. Annals of oncology: official journal of the European Society for Medical Oncology. 2012; 23: 664-70.

25. Fields RC, Strong VE, Gonen M, Goodman KA, Rizk NP, Kelsen DP, et al. Recurrence and survival after pathologic complete response to preoperative therapy followed by surgery for gastric or gastrooesophageal adenocarcinoma. British journal of cancer. 2011; 104: 1840-7.

26. Mokdad AA, Yopp AC, Polanco PM, Mansour JC, Reznik SI, Heitjan DF, et al. Adjuvant Chemotherapy vs Postoperative Observation Following Preoperative Chemoradiotherapy and Resection in Gastroesophageal Cancer: A Propensity Score-Matched Analysis. JAMA oncology. 2018; 4: 31-8.

27. Mokdad AA, Ali A, Yopp AC, Polanco PM, Nassour I, Mansour JC, et al. Adoption of evidence-based novel therapies in the treatment of gastric cancer: A national observational study. Cancer. 2018; 124: 1122-31.

28. Cancer Genome Atlas Research N. Comprehensive molecular characterization of gastric adenocarcinoma. Nature. 2014; 513: 202-9.

29. Sohn BH, Hwang JE, Jang HJ, Lee HS, Oh SC, Shim JJ, et al. Clinical Significance of Four Molecular Subtypes of Gastric Cancer Identified by The Cancer Genome Atlas Project. Clinical cancer research: an official journal of the American Association for Cancer Research. 2017; [Epub ahead of print].

30. Ronellenfitsch U, Schwarzbach M, Hofheinz R, Kienle P, Kieser M, Slanger TE, et al. Preoperative chemo(radio)therapy versus primary surgery for gastroesophageal adenocarcinoma: systematic review with meta-analysis combining individual patient and aggregate data. European journal of cancer. 2013; 49: 3149-58. 